\title{
A complete Heyting algebra whose Scott space is non-sober
}

\author{
$\mathrm{Xu}$ Xiaoquan, Xi Xiaoyong, and Zhao Dongsheng
}

\begin{abstract}
We prove that (1) for any complete lattice $L$, the set $\mathcal{D}(L)$ of all nonempty saturated compact subsets of the Scott space of $L$ is a complete Heyting algebra (with the reverse inclusion order); and (2) if the Scott space of a complete lattice $L$ is non-sober, then the Scott space of $\mathcal{D}(L)$ is non-sober. Using these results and the Isbell's example of a non-sober complete lattice, we deduce that there is a complete Heyting algebra whose Scott space is non-sober, thus give a positive answer to a problem posed by Jung. We will also prove that a $T_{0}$ space is well-filtered iff its upper space (the set $\mathcal{D}(X)$ of all nonempty saturated compact subsets of $X$ equipped with the upper Vietoris topology) is well-filtered, which answers another open problem.
\end{abstract}

Sobriety and well-filteredness are two of the most important properties for non-Hausdorff topological spaces. The Scott space of every domain (continuous directed complete posets) is sober. Johnstone [8] gave the first example of a dcpo whose Scott space is non-sober. Soon after that, Isbell [6] constructed a complete lattice whose Scott space is non-sober. The general problem in this line is whether each object in a classic class of lattices has a sober Scott space. The Isbell's non-sober complete lattice is not distributive. Thus recently Jung asked whether there is a distributive complete lattice whose Scott space is non-sober. In this paper we shall give a positive answer to Jung's problem. The main structure we shall use is the poset $\mathcal{D}(X)$ of all nonempty saturated compact subsets of a topological space $X$ equipped with the reverse inclusion order. We first show that for any complete lattice $L$, the poset $\mathcal{D}(L)$ of all nonempty saturated compact subsets of the Scott space of $L$ is a complete Heyting algebra. Then we prove that for a certain type $T_{0}$ spaces $X$, if $X$ is non-sober, then the Scott space of $\mathcal{D}(X)$ is non-sober. An immediate conclusion is that for any complete lattice $L$,

2000 Mathematics Subject Classification. 06B35, 06B30, 54A05.

Key words and phrases. Scott topology; sober space; well-filtered space; upper space. 
if the Scott space of $L$ is non-sober, then the Scott space of the complete Heyting algebra $\mathcal{D}(L)$ is non-sober. Taking $L$ to be the Isbell's example, we obtain a complete Heyting algebra whose Scott space is non-sober, thus answer Jung's problem.

Heckmann and Keimel 4 proved that a space $X$ is sober if and only if the upper space $\mathcal{D}(X)$ of $X$ is sober. In [12], Xi and Zhao proved that a space $X$ is well-filtered iff its upper space $\mathcal{D}(X)$ is a d-space. But, it is still not known, as pointed out in [12], whether it is true that a space is well-filtered if and only if the upper space of $X$ is well-filtered. In the last part of this paper we will give a positive answer to this problem.

\section{Preliminaries}

A complete Heyting algebra is a complete lattice $L$ satisfying the following infinite distributive law:

$$
a \wedge \bigvee\left\{a_{i}: i \in I\right\}=\bigvee\left\{a \wedge a_{i}: i \in I\right\}
$$

for any $a \in L$ and $\left\{a_{i}: i \in I\right\} \subseteq L$. Such a complete lattice is also called a frame. Apparently every complete Heyting algebra is a distributive complete lattice.

An element $p$ of a meet-semilattice $S$ is a prime element if for any $a, b \in$ $S, a \wedge b \leq p$ implies $a \leq p$ or $b \leq p$. A frame (or complete Heyting algebra) $A$ is called spacial if every element of $A$ can be expressed as a meet of prime elements. It is well-known that a complete lattice $L$ is a spacial frame iff it is isomorphic to the lattice of all open subsets of some topological space (cf. [9]).

A subset $U$ of a poset $(P, \leq)$ is $S$ cott open if i) $U$ is an upper set (that is, $U=\uparrow U=\{x \in P: y \leq x$ for some $y \in U\}$ ), and ii) for any directed subset $D \subseteq P, \bigvee D \in U$ implies $D \cap U \neq \emptyset$ whenever $\bigvee D$ exists. All Scott open sets of a poset $P$ form a topology on $P$, denoted by $\sigma(P)$ and called the $S$ cott topology on $P$. The space $(P, \sigma(P))$ is denoted by $\Sigma P$, called the $S$ cott space of $P$.

A poset is called a directed complete poset (dcpo, for short) if every directed subset of the poset has a supremum. For more about Scott topology and dcpos, see [1] 2].

A subset $A$ of a topological space is called saturated if $A$ equals the intersection of all open sets containing it. A $T_{0}$ space $X$ is well-filtered if for any open set $U$ and filtered family $\mathcal{F}$ of saturated compact subsets of $X$, $\bigcap \mathcal{F} \subseteq U$ implies $F \subseteq U$ for some $F \in \mathcal{F}$.

A subset $A$ of a space $X$ is irreducible if for any closed subsets $F_{1}, F_{2}$ of $X, F \subseteq F_{1} \cup F_{2}$ implies $F \subseteq F_{1}$ or $F \subseteq F_{2}$. Obviously, the closure of every singleton set is irreducible. A space $X$ is called sober if every irreducible 
closed set of $X$ is the closure of a unique singleton set. It is well known that every sober space is well-filtered. Jonstone [8 constructed the first example of a dcpo whose Scott space is non-sober. Isbell [6] constructed a complete lattice whose Scott space is non-sober. Kou [5] gave the first example of a dcpo whose Scott space is well-filtered but non-sober.

The specialization order $\leq_{\tau}$ on a $T_{0}$ space $(X, \tau)$ is defined by $x \leq_{\tau} y$ iff $x \in c l(\{y\})$, where $c l(\{y\})$ is the closure of set $\{y\}$. A space $(X, \tau)$ is called a $d$-space (or monotone convergence space) if $\left(X, \leq_{\tau}\right)$ is a dcpo and $\tau \subseteq \sigma\left(\left(X, \leq_{\tau}\right)\right)($ cf. [1] ).

\section{The existence of a complete Heyting algebra whose Scott space is non-sober}

For any topological space $X$, following Heckmann and Keimel [4] we shall use $\mathcal{D}(X)$ to denote the set of all nonempty compact saturated subsets of $X$. The upper Vietoris topology on $\mathcal{D}(X)$ is the topology that has $\{\square U$ : $U \in \mathcal{O}(X)\}$ as a base, where $\square U=\{K \in \mathcal{D}(X): K \subseteq U\}$. The set $\mathcal{D}(X)$ equipped with the upper Vietoris topology is called the Smyth power space or upper space of $X$ (cf. [4] [10]).

The specialization order on the upper space $\mathcal{D}(X)$ is the reverse inclusion order $\supseteq$. In what follows, the partial order on $\mathcal{D}(X)$ we will concern is just the reverse inclusion order.

For a poset $P$, we shall use $\mathcal{D}(P)$ to denote the poset of all nonempty compact saturated subsets of the Scott space $(P, \sigma(P))$.

A space $X$ is called coherent if the intersection of any two compact saturated subsets in $X$ is compact.

Lemma 1. For any complete lattice $L, \mathcal{D}(L)$ is a complete Heyting algebra.

Proof. The Scott space $\Sigma L$ of $L$ is well-filtered by Xi and Lawson[11, and is coherent by Jia and Jung [7]. We now show that the poset $\mathcal{D}(L)$ is a complete Heyting algebra.

(i) From that $(L, \sigma(L))$ is well-filtered, it follows that $\mathcal{D}(L)$ is closed under filtered intersections, thus it is a dcpo, in which the infimum of a directed subsets $\mathcal{K}$ of $\mathcal{D}(L)$ is the intersection of $\mathcal{K}$.

(ii) Also $\Sigma L$ is coherent and every member of $\mathcal{D}(L)$ contains the top element $1_{L}$ of $L$, so the intersection $K_{1} \cap K_{2}$ of any two members $K_{1}, K_{2}$ of $\mathcal{D}(L)$ is again a member of $\mathcal{D}(L)$, which equals the join $K_{1} \vee K_{2}$ of $K_{1}$ and $K_{2}$ in $\mathcal{D}(L)$. Also $\mathcal{D}(L)$ has $L$ as the least element, and $\left\{1_{L}\right\}$ as the top element. It follows that $\mathcal{D}(L)$ is both a dcpo and a join semilattice, and has a least element. Therefore $\mathcal{D}(L)$ is a complete lattice. In addition, for any $K_{1}, K_{2} \in \mathcal{D}(L)$, the meet $K_{1} \wedge K_{2}$ of $K_{1}, K_{2}$ in $\mathcal{D}(L)$ clearly equals $K_{1} \cup K_{2}$. 
Now for any subfamily $\left\{K_{i}: i \in I\right\} \subseteq \mathcal{D}(L)$, from (i) and (ii) we can deduce that

$$
\bigvee\left\{K_{i}: i \in I\right\}=\bigcap\left\{K_{i}: i \in I\right\} .
$$

Then for any $K \in \mathcal{D}(L)$ and $\left\{K_{i}: i \in I\right\} \subseteq \mathcal{D}(L)$, we have

$$
\begin{aligned}
K \wedge \bigvee_{i \in I} K_{i} & =K \cup\left(\bigcap_{i \in I} K_{i}\right) \\
& =\bigcap_{1 \in I}\left(K \cup K_{i}\right) \\
& =\bigvee_{i \in I}\left(K \wedge K_{i}\right) .
\end{aligned}
$$

Hence $\mathcal{D}(L)$ is a complete Heyting algebra.

For any $T_{0}$ space $(X, \tau)$, let $\xi_{X}: X \rightarrow \mathcal{D}(X)$ be the canonical mapping given by

$$
\xi_{X}(x)=\uparrow x=\left\{y \in X: x \leq_{\tau} y\right\} .
$$

It is easy to see that $\xi_{X}:\left(X, \leq_{\tau}\right) \longrightarrow(\mathcal{D}(D), \supseteq)$ is an order embedding.

To emphasize the codomain, we shall use $\xi_{X}^{\sigma}$ to denote the corresponding mapping $\xi_{X}^{\sigma}:(X, \tau) \longrightarrow(\mathcal{D}(X), \sigma(\mathcal{D}(X)))$, where $\xi_{X}^{\sigma}(x)=\uparrow x$ for each $x \in X$.

TheOREM 1. Let $X$ be a $T_{0}$ space such that

(i) the upper Vietoris topology on $\mathcal{D}(X)$ is contained in $\sigma(\mathcal{D}(X)$ ) (that $i s$, the upper Vietoris topology is weaker than the Scott topology);

(ii) the mapping $\xi_{X}^{\sigma}:(X, \tau) \longrightarrow(\mathcal{D}(X), \sigma(\mathcal{D}(X)))$ is continuous; and

(iii) $(\mathcal{D}(X), \sigma(\mathcal{D}(X)))$ is sober.

Then $X$ is sober.

Proof. Let $F$ be a nonempty closed irreducible subset of $X$. Then, as $\xi_{X}^{\sigma}$ is continuous, $\xi_{X}^{\sigma}(F)$ is an irreducible subset of $(\mathcal{D}(X), \sigma(\mathcal{D}(X)))$. Therefore, there exists $K \in \mathcal{D}(X)$ such that

$$
c l_{\sigma(\mathcal{D}(X))}\left(\xi_{X}^{\sigma}(F)\right)=\downarrow_{\mathcal{D}(X)} K(=\{A \in \mathcal{D}(X): K \subseteq A\}),
$$

where $c l_{\sigma(\mathcal{D}(X))}$ is the closure operator in the Scott space $(\mathcal{D}(X), \sigma(\mathcal{D}(X)))$.

Claim 1. Every element of $K$ is an upper bound of $F$ in the poset $\left(X, \leq_{\tau}\right)$.

In fact, let $k \in K$ and $x \in F$. Then $\uparrow k \in \mathcal{D}(X)$ and $\uparrow k \subseteq K$. In addition, $\uparrow x=\xi_{X}^{\sigma}(x) \in \downarrow_{\mathcal{D}(X)} K$, so $\uparrow x \supseteq K$. Hence $\uparrow x \supseteq K \supseteq \uparrow k$, which implies $x \leq_{\tau} k$.

Claim 2. $K$ has a least element.

If, on the contrary, for each $k \in K$, there is $s(k) \in K$ such that $k \mathbb{Z}_{\tau} s(k)$. Then

$$
K \subseteq \bigcup\{X \backslash \downarrow s(k): k \in K\} .
$$

Thus $K \in \square \bigcup\{X \backslash \downarrow s(k): k \in K\} \in \sigma(\mathcal{D}(X))$ (by the assumption (i) in Theorem 1), implying

$$
K \in c l_{\sigma(\mathcal{D}(X))}\left(\xi_{X}^{\sigma}(F)\right) \cap \bigcup\{X \backslash \downarrow s(k): k \in K\}
$$


A COMPLETE HYTING ALGEBRA WHOSE SCOTT SPACE IS NON-SOBER

Hence

$$
c l_{\sigma(\mathcal{D}(X))}\left(\xi_{X}^{\sigma}(F)\right) \cap \square \bigcup\{X \backslash \downarrow s(k): k \in K\} \neq \emptyset .
$$

Therefore $\xi_{X}^{\sigma}(F) \cap \square \bigcup\{X \backslash \downarrow s(k): k \in K\} \neq \emptyset$. Hence there exists $y \in F$ with $\uparrow y \subseteq \bigcup\{X \backslash \downarrow s(k): k \in K\}$. It follows that $y \not \mathbb{Z}_{\tau} s(k)$ for some $s(k) \in K$. But this contradicts Claim 1 (every element of $K$ is an upper bound of $F$ ).

Therefore $K$ has a least element, say $s$. Then $K=\uparrow s$.

Claim 3. $F=c l_{X}(\{s\})$. As $s$ is an upper bound of $F$ and $F$ is closed, we only need to confirm that $s \in F$.

Assume, on the contrary, that $s \notin F$. Then $K \subseteq X \backslash F$, so

$$
K \in c l_{\sigma(\mathcal{D}(X))}\left(\xi_{X}^{\sigma}(F)\right) \cap \square(X \backslash F)
$$

and $\square(X \backslash F) \in \sigma(\mathcal{D}(X))$.

Therefore $\xi_{X}^{\sigma}(F) \cap \square(X \backslash F) \neq \emptyset$, which is impossible.

Hence $s \in F$, thus $F=\downarrow s=c l_{X}(\{s\})$.

All these together show that $(X, \tau)$ is sober.

REMARK 1. (1) For every $T_{0}$ space $X$, the mapping $\xi_{X}: X \longrightarrow \mathcal{D}(X)$ (the upper space of $X$ ) is a topological embedding (cf. [4]).

(2) For any poset $P$, the mapping

$$
\xi_{P}^{\sigma}:(P, \sigma(P)) \longrightarrow(\mathcal{D}(P), \sigma(\mathcal{D}(P)))
$$

is continuous, i.e., it preserves all existing suprema.

(3) Every well-filtered space is a d-space.

(4) A $T_{0}$ space $X$ is well-filtered iff $\mathcal{D}(X)$ is a dcpo and the upper Vietoris topology on $\mathcal{D}(X)$ is contained in $\sigma(\mathcal{D}(D)$ ) (equivalently, the upper space $\mathcal{D}(X)$ is a d-space) (see [3] 12]). In general, the well-filteredness of $X$ is stronger than the condition that the upper Vietoris topology on $\mathcal{D}(X)$ is contained in $\sigma(\mathcal{D}(D))$.

For example, consider the poset $\mathbb{N}$ of natural numbers with the usual order. Then every element in $\mathbb{N}$ is compact and so $\mathbb{N}$ is an algebraic poset. Hence $\Sigma \mathbb{N}(\sigma(\mathbb{N})$ equals the Alexandroff topology on $\mathbb{N})$ is locally compact and $\mathcal{D}(\mathbb{N})=\{\uparrow n: n \in \mathbb{N}\}$, which is isomorphic to $\mathbb{N}$. Therefore $\mathcal{D}(\mathbb{N})$ is not a dcpo. Now we have that the upper Vietoris topology on $\mathcal{D}(\mathbb{N})$ equals the Scott topology $\sigma(\mathcal{D}(\mathbb{N})$ ) (and also equals the Alexandroff topology on $\mathcal{D}(\mathbb{N}))$. But $\Sigma \mathbb{N}$ is not well-filtered.

ExAmPLE 1 . Let $X$ be any non-countable set and $\tau$ be the co-countable topology on $X$. Then $(X, \tau)$ is a $T_{1}$ space. Clearly, the nonempty compact (saturated) subsets of $(X, \tau)$ are exactly the nonempty finite subsets of $X$, that is, $\mathcal{D}(X)=\{F: F$ is a nonempty finite subset of $X\}$. Every directed subset $\mathcal{E}$ of $\mathcal{D}(X)$ has a largest element (which is the intersection of $\mathcal{E}$ ), so $(X, \tau)$ is well-filtered but non-sober ( $X$ is an irreducible closed set but not the closure of any singleton set). Clearly $\mathcal{D}(X)$ is a dcpo and every element 
in $\mathcal{D}(X)$ is compact. Hence $\mathcal{D}(X)$ is an algebraic domain and $\sigma(\mathcal{D}(X))$ (which equals the Alexandroff topology on $\sigma(\mathcal{D}(X))$ ) is sober. For $(X, \tau)$, the conditions (i) and (iii) in Theorem 1 are satisfied, but the assumption (ii) does not hold. In this case, the sobriety of $(\mathcal{D}(X), \sigma(\mathcal{D}(X)))$ does not imply the sobriety of $(X, \tau)$.

By Remark 1(4), Theorem 1 can be restated as the following one.

TheOREM 2. Let $(X, \tau)$ be a $T_{0}$ space such that

(i) $X$ is well-filtered;

(ii) the mapping $\xi_{X}^{\sigma}:(X, \tau) \longrightarrow(\mathcal{D}(X), \sigma(\mathcal{D}(X)))$ is continuous; and

(iii) $(\mathcal{D}(X), \sigma(\mathcal{D}(X)))$ is sober.

Then $X$ is sober.

By Theorem 2 and Remark 1, we deduce the following.

Corollary 1. For a dcpo $P$, if $(P, \sigma(P))$ is well-filtered and $(\mathcal{D}(P)$, $\sigma(\mathcal{D}(P)))$ is sober (equivalently, the upper space $\mathcal{D}(P)$ is a d-space and $(\mathcal{D}(P), \sigma(\mathcal{D}(P)))$ is sober $)$, then $(P, \sigma(P))$ is sober.

By $\mathrm{Xi}$ and Lawson [11, for any complete lattice $L,(L, \sigma(L))$ is wellfiltered, hence the upper Vietoris topology on $\mathcal{D}(L)$ is contained in $\sigma(L)$. Now applying Corollary 1, we obtain the following.

THEOREM 3. For any complete lattice $L$, if $(L, \sigma(L))$ is non-sober, then $(\mathcal{D}(L), \sigma(\mathcal{D}(L)))$ is non-sober.

Now we are ready to answer Jung's problem mentioned in the introduction.

ExAmple 2. In [6], Isbell constructed a complete lattice whose Scott topology is non-sober, thus answered a question posed by Johnstone in $[\mathbf{8}]$. The Isbell's complete lattice is not distributive. In one of his recent talk in Singapore, Achim Jung asked whether there is a distributive complete lattice whose Scott topology is non-sober. We now can give a positive answer to this problem. Take $M$ be the complete lattice constructed by Isbell and let $L=\mathcal{D}(M)$. Then by Lemma 1, $L$ is a complete Heyting algebra. Since the Scott space of $M$ is non-sober, by Theorem 3 , the Scott space of $L$ is non-sober. Hence $L$ is a complete Heyting algebra whose Scott space is non-sober.

REMARK 2. For any $T_{0}$ space $(X, \tau)$, the poset $(\mathcal{D}(X), \supseteq)$ is a meetsemilattice, where the meet of $K_{1}, K_{2} \in \mathcal{D}(X)$ equals $K_{1} \cup K_{2}$. Then clearly every principle filter $\uparrow x=\left\{y \in X: x \leq_{\tau} y\right\}$ is a prime element of $\mathcal{D}(X)$. In addition, for any $K \in \mathcal{D}(X)$,

$$
K=\bigwedge\{\uparrow x: x \in K\},
$$


A COMPLETE HYTING ALGEBRA WHOSE SCOTT SPACE IS NON-SOBER

showing that every element of $\mathcal{D}(X)$ can be expressed as a meet of prime elements. Hence by Lemma 1, $(\mathcal{D}(L), \supseteq)$ is actually a spacial frame for any complete lattice $L$ (see 9 for more about spatial frames). Thus the nonsober complete Heyting algebra $L$ obtained in Example 2 is also a spacial frame.

\section{Well-filteredness of upper spaces}

In this section, the symbol $\mathcal{D}(X)$ will denote the upper space of topological space $X$.

In [4], it is proved that a space $X$ is sober iff the upper space $\mathcal{D}(X)$ is sober. In 12, it is proved that a $T_{0}$ space $X$ is well-filtered if and only if its upper space is a d-space. As remarked in [12], it is still not known the answer to the following problem: Must the upper space $\mathcal{D}(X)$ be wellfiltered if $X$ is well-filtered?

We now give a positive answer to the above problem.

Lemma 2. ([4]) Let $X$ be a topological space and $\mathcal{A}$ an irreducible subset of the upper space $\mathcal{D}(X)$. Then every closed set $C \subseteq X$ that meets all members of $\mathcal{A}$ contains an irreducible closed subset $A$ that still meets all members of $\mathcal{A}$.

REMARK 3. The irreducible closed set $A$ in Lemma 2 can be take as a minimal one: if $A^{\prime}$ is a closed set, $A^{\prime} \subseteq A$ and meets all members of $\mathcal{A}$, then $A^{\prime}=A$ (see the proof of Lemma 3.1 in [4]).

The following result can be verified straightforwardly (see e.g. [9, page 128] or the proof of Lemma 3.1 in [7]).

LEMma 3. If $\mathcal{K} \subseteq \mathcal{D}(X)$ is a nonempty compact subset of $\mathcal{D}(X)$, then $\bigcup \mathcal{K} \in \mathcal{D}(X)$.

ThEOREM 4. A topological space $X$ is well-filtered iff its upper space $\mathcal{D}(X)$ is well-filtered.

Proof. We only need to show that if $X$ is well-filtered, then so is $\mathcal{D}(X)$. Let $\left\{\mathcal{K}_{t}: t \in T\right\}$ be a filtered family of saturated compact subsets of $\mathcal{D}(X)$, $\mathcal{U}=\bigcup\left\{\square U_{i}: i \in I\right\}$ an open set of $\mathcal{D}(X)$ such that

$$
\bigcap\left\{\mathcal{K}_{t}: t \in T\right\} \subseteq \mathcal{U}
$$

Suppose that $\mathcal{K}_{t} \nsubseteq \mathcal{U}$ for all $t$, that is, $\mathcal{K}_{t} \cap(\mathcal{D}(X) \backslash \mathcal{U}) \neq \emptyset$. Then as $\left\{\mathcal{K}_{t}: t \in T\right\}$ is an irreducible subset of the space $\mathcal{D}(X)$, by Lemma 2 and Remark 3 , there is a minimal closed irreducible subset $\mathcal{C} \subseteq \mathcal{D}(X) \backslash \mathcal{U}$ that meets every $\mathcal{K}_{t}(t \in T)$.

Let $\mathcal{C}=\bigcap\left\{\diamond C_{j}: j \in J\right\}$, where each $C_{j}$ is a closed subset of $X$ and $\diamond C_{j}=\left\{F \in \mathcal{D}(X): F \cap C_{j} \neq \emptyset\right\}$. For each $t \in T$, let $K_{t}=\bigcup\left(\mathcal{K}_{t} \cap \mathcal{C}\right)$. As $\mathcal{K}_{t} \cap \mathcal{C}$ is 
nonempty and compact in $\mathcal{D}(X)$, by Lemma 3 we have that $K_{t} \in \mathcal{D}(X)$. Also $\left\{K_{t}: t \in T\right\}$ is a filtered family of member of $\mathcal{D}(X)$, thus $K=\bigcap\left\{K_{t}: t \in T\right\}$ is a member of $\mathcal{C}(X)$ because $X$ is well-filtered.

Claim 1. $K \notin \mathcal{U}$.

Assume, on the contrary, that $K \in \mathcal{U}$. Then $K=\bigcap\left\{K_{t}: t \in T\right\} \subseteq U_{i}$ for some $i \in I$. Then, as $X$ is well-filtered, $K_{t} \subseteq U_{i}$ holds for some $t \in T$. Then $\mathcal{K}_{t} \cap \mathcal{C} \subseteq \square U_{i} \subseteq \mathcal{U}$, contradicting $\mathcal{C} \subseteq \mathcal{D}(X) \backslash \mathcal{U}$.

Claim 2. $K \in \bigcap\left\{\uparrow_{\mathcal{D}(X)}\left(\mathcal{K}_{t} \cap \mathcal{C}\right): t \in T\right\}$.

Suppose, on the contrary, that $K \notin \bigcap\left\{\uparrow_{\mathcal{D}(X)}\left(\mathcal{K}_{t} \cap \mathcal{C}\right): t \in T\right\}$. Then there is $t_{0} \in T$ such that $K \notin \uparrow \mathcal{D}(X)\left(\mathcal{K}_{t_{0}} \cap \mathcal{C}\right)$. Thus, for any $G \in \mathcal{K}_{t_{0}} \cap \mathcal{C}$, there exists $e(G) \in K \backslash G$. Then $G \cap \downarrow e(G)=\emptyset$ (Note that $G$ is a saturated compact set). Now for any $G \in \mathcal{K}_{t_{0}} \cap \mathcal{C}$ and any $t \in T$, since $e(G) \in K$ (so $\left.e(G) \in K_{t}\right)$, we have $e(G) \in \bigcup\left(\mathcal{K}_{t} \cap \mathcal{C}\right)$. Thus there exists $H_{t} \in \mathcal{K}_{t} \cap \mathcal{C}$ such that $e(G) \in H_{t}$, implying

It follows that

$$
H_{t} \in \mathcal{K}_{t} \cap \mathcal{C} \cap \diamond(\downarrow e(G)) .
$$

$$
\mathcal{K}_{t} \cap \mathcal{C} \cap \diamond(\downarrow e(G)) \neq \emptyset, \text { for all } t \in T .
$$

By the minimality of $\mathcal{C}$, we have $\mathcal{C} \cap \diamond(\downarrow e(G))=\mathcal{C}$, which implies $\mathcal{C} \subseteq \diamond(\downarrow$ $e(G))$.

Therefore $\mathcal{C} \subseteq \bigcap\left\{\diamond(\downarrow e(G)): G \in \mathcal{K}_{t_{0}} \cap \mathcal{C}\right\}$. Note that for any $G \in \mathcal{K}_{t_{0}} \cap \mathcal{C}, G \notin \diamond$ $(\downarrow e(G))$. Hence

$$
\emptyset \neq \mathcal{K}_{t_{0}} \cap \mathcal{C}=\mathcal{K}_{t_{0}} \cap \mathcal{C} \cap \bigcap\left\{\diamond(\downarrow e(G)): G \in \mathcal{K}_{t_{0}} \cap \mathcal{C}\right\}=\emptyset .
$$

This contradiction confirms Claim 2.

Now $K \in \bigcap\left\{\uparrow_{\mathcal{D}(X)}\left(\mathcal{K}_{t} \cap \mathcal{C}\right): t \in T\right\} \subseteq \bigcap\left\{\mathcal{K}_{t}: t \in T\right\} \subseteq \mathcal{U}$, which implies $K \in \mathcal{U}$. But this contradicts Claim 1.

All these together deduce that there must be some $t_{0} \in T$ such that $\mathcal{K}_{t_{0}} \subseteq \mathcal{U}$

Hence $\mathcal{D}(X)$ is well-filtered. The proof is completed.

The following result collects some of the equivalent conditions for a space to be well-filtered.

Theorem 5. For any $T_{0}$ space $X$, the following statements are equivalent:

(1) $X$ is well-filtered.

(2) The upper space $\mathcal{D}(X)$ of $X$ is a d-space.

(3) The upper space $\mathcal{D}(X)$ of $X$ is well-filtered.

Acknowledgement (1) The first author is sponsored by NSFC (11661057), the Ganpo 555 project for leading talent of Jiangxi Provence and the Natural Science Foundation of Jiangxi Provence, China (20161BAB2061004). 
A COMPLETE HYTING ALGEBRA WHOSE SCOTT SPACE IS NON-SOBER

(2) The second author is sponsored by NSFC (11361028, 61300153, 11671008, 11701500, 11626207) and NSF Project of Jiangsu Province, China (BK20170483).

(3) The third author is sponsored by NIE AcRF (RI 3/16 ZDS).

\section{References}

[1] G. Gierz, K. Hofmann, K. Keimel, J. Lawson, M. Mislove, D. Scott, Continuous Lattices and Domains, Cambridge University Press, 2003.

[2] J. Goubault-Larrecq, Non-Hausdorff topology and Domain Theory, Cambridge University Press, 2013.

[3] R. Heckmann, An upper power domain construction in terms of strongly compact sets, in: Lecture Notes in Computer Science, vol. 598, Springer, Berlin Heidelberg New York, 1992, pp. 272-293.

[4] R. Heckmann, K. Keimel, Quasicontinuous domains and the Smyth powerdomain, Electronic Notes in Theor. Comp. Sci. 298 (2013) 215-232.

[5] H. Kou, $U_{k}$-admitting dcpos need not be sober, in: Domains and Processes, Semantic Structure on Domain Theory, vol. 1, Kluwer, 2001, pp. 41-50.

[6] J. Isbell, Completion of a construction of Johnstone, Proc. Amer. Math. Soci. 85 (1982) 333-334.

[7] X. Jia, A. Jung, A note on coherence of dcpos, Topol. Appl. 209 (2016) 235-238.

[8] P. Johnstone, Scott is not always sober, in: Continuous Lattices, Lecture Notes in Math., vol. 871, Springer-Verlag, 1981, pp. 282-283.

[9] P. Johnstone, Stone Spaces, Cambridge University Press, 1982.

[10] A. Schalk, Algebras for Generalized Power Constructions, PhD Thesis, Technische Hochschule Darmstadt, 1993.

[11] X. Xi, J. Lawson, On well-filtered spaces and ordered sets, Topol. Appl. 228 (2017) 139-144.

[12] X. Xi, D. Zhao, Well-filtered spaces and their dcpo models, Math. Struct. Comput. Sci. 27 (2017) 507-515.

School of Mathematics and Statistics, Minnan Normal University, FuJiAn, Zhangzhou 363000, P.R. China

E-mail address: xiqxu2002@163.com

School of mathematics and Statistics, Jiangsu Normal University, Jiangsu, Xuzhou, P.R. China

E-mail address: littlebrook@jsnu.edu.cn

Mathematics and Mathematics Education, National Institute of Education Singapore, Nanyang Technological University, 1 Nanyang Walk, SingAPORE 637616

E-mail address: dongsheng.zhao@nie.edu.sg 